\title{
Towards New Integrated Information and Communication Infrastructures in E-Health. Examples from Cardiology
}

\author{
J Fayn, C Ghedira, D Telisson, H Atoui, J Placide, L Simon-Chautemps, P Chevalier, P Rubel \\ INSERM ERM107 Methodologies and Information Processing in Cardiology, Lyon, France
}

\begin{abstract}
An operational e-health infrastructure including pervasive services and remote distributed applications servers in the cardiology domain is presented. Based on the implementation of previously reported software components building blocks, developed in particular within the frame of European projects, the architecture promotes and facilitates the use of advanced methods of quantitative electrocardiology. The expected outcome is the improvement of the early detection of cardiac diseases, of the patients follow-up and the enhancement of the citizens quality of life.
\end{abstract}

\section{Introduction}

A new era of virtual telemedicine focusing on the integration of the new information and communication technologies and of various building blocks of business software components based on the past knowledge is becoming reality. The development of such a new type of integrated infrastructure is especially relevant in the cardiology domain, mainly because of the potential clinical scenarios of use and of the possible impact on the improvement of the quality of life, and because the domain benefits from a large experience in automated, reusable specialized applications.

The infrastructure we present in this paper is based on information and communication tools built within the framework of previous European projects, in particular SCP-ECG, OEDIPE and EPI-MEDICS, and on the French TIISSAD project results [1-4]. We will also address different scenarios of use of telematic tools and pervasive services in various environments, by the confirmed health professional as well as by the citizen, including routine consultations in cardiology, home-care, self-care and emergency tele-expertise.

\section{Towards a new integrated e-health infrastructure in cardiology}

An e-health infrastructure should comply today with various clinical settings such as the follow-up of patients with cardiac diseases, of pregnancy and of elderly people. Its design should be mainly focused on the sharing of distributed resources, including web services, grid-type architectures and application servers, between the various involved actors. The latter are medical professionals, specialized or not in cardiology such as the general practitioners, working in various health-care providers organizational contexts, but also the patient himself, his family and any medical auxiliary. The available resources should be generic services, compliant with all medical specialities such as the retrieval of a medical record or the delivery of a prescription everywhere at any time, and integrated specialized cardiology services such as ECG processing and decision-making.

We present in the following a clinical use-case of such an architecture involving the access to different distributed resources, and then a set of complementary building blocks providing some of the main relevant basic services and composed of software components that have already been developed and implemented. We finally present an operational implementation of the overall design of such an infrastructure.

\subsection{Clinical scenario description}

Let us take the example of a working person who has suddenly a severe chest pain and falls in a faint when walking in the street. The closest emergency centre is called, and an ambulance is coming to take care of this patient. The emergency physician collects clinical data and records an ECG from this patient. As soon as the physician suspects a heart attack, he should send to the emergency department, where the patient will be admitted, a brief mobile medical record (MMR) describing the clinical status of the patient and including the recorded ECG. The e-health record is built at bedside and sent during the transportation of the patient in the ambulance. Before the arrival of the patient, a physician of the emergency department will display the MMR with the ECG, and will prepare the transfer of the patient to the ICU, and for an angioplasty if needed. Until that time, the patient will have several serial ECGs to assess his clinical status. Some days later, the patient could have a coronary bypass surgery. Then, after being discharged from the hospital, the patient will possibly stay some time in a specialized, not necessary medical, institution, and then at home for recovery. During this convalescence 
period, the patient's follow-up will be performed by various health professionals such as a referral physician, a GP, as well as by cardiologists from the hospital in routine consultation.

At each step, at the place where the patient will be, clinical data and different examinations such as ECGs will be collected. All this information should be shared for display and update by the various involved medical actors. When he returns at home, the patient should also be able to collect himself clinical data with blood pressure and ECG records, and to send a MMR to his referral physician, either periodically, according to a predefined protocol, for control and/or for medical treatment adjustment, or voluntarily in case of a pain event occurrence. The referral cardiologists should be able to retrieve all these data, including ECG signals and images, and to display trend profiles of some relevant measurements, such as blood pressure and ST segment deviations for instance. They should have also access to additional computerized processing facilities in order to improve their diagnosis accuracy, by performing serial ECG analysis [5] and by using decision-making methods such as artificial neural networks [6].

\subsection{Software architecture building blocks}

The first main building block that is necessary for the set-up of an e-health infrastructure is the existence of standard communications protocols for the exchange of heterogeneous data. In the cardiology domain, this has already been almost solved thanks to the development of the European Norm SCP-ECG for the exchange of ECG signals [1], of the DICOM Standard for medical images, and of the XML meta-language enabling the concrete structured representation of demographic and clinical information, including quantitative and qualitative data, and able to encapsulate binary files such as SCP-ECG or DICOM files. All these standards are compliant with TCP/IP based communication technologies. In particular, SCP-ECG enables the syntactic and semantic representation of digital ECG signals and measurements, and of all the corresponding metadata, such as the recording conditions, that are necessary from the technical and clinical context points of view for their exploitation, if needed their reanalysis, and their medical interpretation.

The second main building block is the existence of reference core database models for data storage and retrieval that may be easily implemented in the most widely and currently used relational database management systems. Let us take as an example the OEDIPE core data model that has been designed to support the storage of serial SCP-ECG compliant data, as well as the storage of their analysis and interpretation results from multiple programs [7]. SCP-ECG original files are also stored as a whole to facilitate their retrieval and to perform further analyses or to forward them, if needed, to another recipient. Furthermore, the OEDIPE core model is endowed with a meta-model for the automatic management and control of serial analyses processes driven by the patients data [8]. In particular, one or several ECG records may be documented as reference examinations for each patient, and linked to descriptors of the patients contextual clinical events. All these features allow an easy and intelligent retrieval of any ECG data only by means of standard SQL queries.

A third main building block aims to provide transparent software facilities to medical centers and physician professionals to take advantage of the opportunities offered by the two previous main building blocks. Data that are stored in a relational database may be easily retrieved by SQL queries, but the reverse process consisting in automatically splitting data contained in a structured file and storing these data in a database, with integrity and coherence control, should also be operational. Some tools are existing for the storage of XML data, provided that they are elementary, numerical or textual, and that they are well structured and semantically well defined. But binary composite data such as encoded ECG signals with their related representation characteristics, such as the Huffman parameters and the fiducial points, require, to be automatically stored and reused, the existence of an intelligent middleware layer of software components. The OEDIPE Project already solved this problem for SCPECG compliant files. The solution that has been developed is based on the design of three meta-models that respectively handle the customized description of any SCP-ECG file, of any target database model and of their relationships that are stored in a rules based data dictionary [2]. The latter may be easily updated by means of a friendly, but secured, user-interface to deal with different hardware and software implementations as well as with the various needs induced by the multiple scenarios of routine clinical use. The SCP to DB ECG middleware is compliant with any instantiations of the SCP-ECG norm, which is an open standard enabling multiple data representations, and with any models of the target database that may differ according to the clinical implementation site.

The fourth main building block consists in a tool able to create, to convey and to display any customized, mobile medical record that may be usable by any actor of the health-care system, including any health professional as well as the patient or citizen himself in case of homecare and self-care. We previously presented a new software architecture, based on the concept of macrodata, that provides all these facilities for tele-expertise applications between general and specialized practitioners from various medical domains [9]. This architecture has now been generalized and applied to support a greater variety of clinical scenarios in pervasive environments. The main concept is to provide an easy-to- 
use and friendly user-interface enabling the creation of a structured, dated and authenticated medical record with a presentation layout that may be completely personalized at the time of its creation by means of utilizations models, and that will remain unchanged whenever it is opened and read, whatever the distribution network. The utilization models may be user and patient specific. An MMR may encapsulate SCP-ECG or DICOM files, and any data files of known format, such as AVI or MPEG files, to quickly document clinical symptoms and observations by the citizen himself. Any mobile medical record is stored as a whole in XML format to facilitate its transportation. Export facilities of attached or embedded specialized files such as SCP-ECG data may be easily setup by means of the middleware software components. This architecture has been instantiated in various usecases, such as the follow-up of cardiac diseased patients, of pregnancy and of elderly people [4], as well as for the flow management control of tele-expertise requests, whatever their initiator: an emergency physician, a general practitioner, a nurse or the patient himself, and for the cooperative management of distributed and shared medical records and of generic services requests such as a prescription for the patient or a summary report on a specific medical treatment or test.

Another main building block is to provide to any physician capabilities of adaptive hypermedia data presentation. The solution recently proposed by Ghedira allows an efficient multimedia data browsing and navigation through heterogeneous medical information [10]. Her HANS method for the development of medical data reviewing support systems is based on three conceptual schemas: the Information Domain, the Navigational and the Actors models, and on two XMLbased application-oriented models related to data structuring and presentation. It handles the capitalization of the users knowledge to improve information access and to facilitate the decision-making process by means of a dynamic and personalized storage of the users preferences and practice in real time.

In addition to the two last generic concepts of data presentation yielding, on one hand, for an accurate and reliable restitution of a customized original medical record and, on the other hand, for an efficient and adaptive navigational retrieval of the relevant data, we may also consider business components for the display of specialized data. Let us take as examples the DICOM viewers that already exist, or the SCP-ECG viewer that has been developed in our institute and is in routine use. The latter supplies the cardiologist with capabilities of interpreting an ECG record, directly on the screen, just by clicking on the ECG file icon, whatever the screen size and resolution. Such a component can be used through any Windows ${ }^{\circledR}$ application. Another example of such a business component that could be embedded in the HANS method for data presentation is the IDEA-based software for Interactive and Dynamic ECG Analysis [11]. The IDEA method takes into account the temporal integration of time or event related multi-source data.

The last main building block is composed of business software components, implementing advanced methods of computerized ECG signal and image processing, and of decision-making. In the field of quantitative electrocardiology, many such relevant applications are existing. As an example, although several well-assessed computerized methods of ECG waves delineation are available, a pre-processing error may occur depending on the signal noise level for instance. It may be therefore useful to correct the pre-processing results and then to perform a re-analysis and the storage of the new results in SCP-ECG format and/or in an ECG database. Another relevant example is to provide the capabilities of performing serial ECG analysis. Comparing a current ECG record with a previous one of the same patient could considerably enhance the ECG diagnostic accuracy [5]. The CAVIAR method is such an example which is able to compare any digital 12-lead or pseudo-orthogonal ECG record with reference to a baseline ECG. Highfrequency ECG analysis can also provide additional useful descriptors. The same yields for computerized decision-making processes, such as artificial neural networks based methods, that could greatly help the physician to interpret ECG data and improve the patients triage in an emergency department for instance [6].

\subsection{An e-health architecture description}

The e-health infrastructure we are proposing is being implemented in routine use in the Lyons area. It includes several sites: the ICU, the emergency department and the routine consultation department of the cardiology hospital, several GPs and town cardiologists offices, the central emergency call centre, the informatics department of all the Lyons hospitals, and virtual and concrete dailylife locations of patients including their place of residence. The hardware infrastructure is composed of standard Windows ${ }^{\circledR}$ compatible PCs, of a TCP-IP based intra-and inter-hospitals network, of Internet connections at the patient's home and at the doctor's offices, and of GPRS communication means for the mobile phones of the patients and of the referral and advisor physicians. Medical equipment includes SCP-ECG compliant electrocardiographs that shall be available for the health professionals at any health provider site, and DICOM compliant imaging systems when medical images are performed. The success of such a pervasive e-health infrastructure implementation relies on the development opportunities that are being offered by the EPI-MEDICS project [3]. The latter supplies the citizens and the patients, as well as any ambulatory health provider, with a Portable ECG Monitor (PEM) having the capabilities of recording and analysing a simplified ECG, of saving the ECG data in an SCP-ECG compliant format, of 
performing in real time an advanced decision-making process based on serial ECG analysis results, if a reference ECG is available, and on some items of the patient's clinical history that have been previously stored in the PEM device. The PEM is then able to make recommendations for the patient decision-making, to automatically launch an emergency request to an alarm server in case of detection of a severe disease, and to transmit via Bluetooth and GPRS an XML message including some clinical items and a standard 12-lead ECG that has been derived from the last recorded 3-lead PEM ECG by means of a patient-specific transformation matrix.

In ambulatory situations, the PEM alone with an appropriate mobile phone is the minimum first-aid kit. At home, in the ambulance, at the doctor's office or in the hospital departments, a PC-based MMR creating and viewing tool should be available, with means for the communication of XML-based MMR messages and a reception software to upload SCP-ECG files from the used ECG device. Only a standard web browser is needed for the PEM because it embeds itself a web server. The software components building blocks, presented above, are then distributed in some of the cardiology departments, with an access to one or several remote service providers application servers where the business components of the last building block might be implemented. Other business components have been or will be installed for the setup and update of the PEM device with the 12-lead transformation matrix and initialization parameters of the decision-making process. Additional services like the flow management process of tele-expertise requests and of PEM alarms are also being implemented in emergency call centers and/or in the informatics department of several hospitals. All these software components will be driven by intelligent mobile agents to facilitate their communication via XML format, to update the meta-databases and for data retrieval.

\section{Conclusion}

The overall e-health operational architecture presented in this paper includes structured and semi-structured data entry facilities, information processing, data storage and retrieval, adaptive hypermedia presentation and decision support. All these steps are driven by intelligent, mobile agents in pervasive computing environments. The expected outcome is the concrete set-up of a global, virtual e-health record for any citizen, easily accessible and sharable by any authorized health-care professional.

\section{Acknowledgements}

This research was supported in part by the Commission of the European Communities, within the frame of its Advanced Informatics in Medicine Program under Project \#A2026 and its Information Society
Technologies under Project IST-2000-26164, and by the national research funding of the French Government within its frame of its Telemedicine and Technologies for Health ACI program under Project 71/99.

\section{References}

[1] CEN/TC251 ENV 1064. Medical Informatics-Standard Communications Protocol for Computer-assisted Electrocar diography. June 1993.

[2] Rubel P, Willems JL, Zywietz C, Fayn J, Assanelli D, Malossi C, Todd S. OEDIPE: Open European Data Interchange and Processing for Electrocardiography. In: Laires MF, Ladeira MJ, Christensen JP, Editors. Health in the New Communications Age. Amsterdam: IOS Press, 1995:313-21.

[3] Rubel P, Gouaux F, Fayn J, Assanelli D, Cuce A, Edenbrandt L, Malossi C. Towards Intelligent and Mobile Systems for Early Detection and Interpretation of Cardiological Syndromes. Computers in Cardiology 2001;28:193-96.

[4] Thomesse JP et al. Integrated information technologies for patients remote follow-up and homecare. In: Patricelli F, Ray PK, Editors. Proceedings of the 3rd International workshop on Enterprise Networking and Computing in Health Care Industry. HEALTHCOM2001. L'Aquila (Italy):SGRR, 2001:3-15.

[5] Fayn J, Rubel P, Forlini, Pahlm O, Wagner G. How to improve the identification of the coronary artery occlusion site in acute myocardial ischemia using computerized ECG analysis methods. Eur Heart J 2003;24 Suppl:604.

[6] Ohlsson M, Ohlin H, Wallerstedt SM, Edenbrandt L. Usefulness of serial electrocardiograms for diagnosis of acute myocardial infarction. Am J Cardiol 2001;88(5):478-81.

[7] Fayn J, Rubel P, Willems JL, Conti L, Reniers R. Design and Implementation Strategies of a Core Database Model for the Storage and Retrieval of Serial ECG Data. In : Murray A, Arzbaecher R, Editors. Computers in Cardiology. Los Alamitos (USA) : IEEE Computer Society Press, 1994:189-92.

[8] Fayn J, Rubel P, Willems JL. Management of Serial ECGs and Control Strategies for the Comparison Process. Meth Inform Med, 1994;33:148-52.

[9] Fayn J, Petitjean F, Selmaoui N, Rubel P. Macro-Data: A New Approach for the Development of Tele-Expertise Applications. Computers in Cardiology 1999;26:41-4.

[10] Ghedira C, Maret P, Fayn J, Rubel P. Adaptative user interface customization through browsing knowledge capitalization. Int J Med Inf 2002;68:219-28.

[11] Fayn J, Conti L, Fareh S, Maison-Blanche P, Nony P, Rubel P. Interactive and Dynamic ECG Analysis. Is it Just an IDEA or a Clinically Relevant Approach ? J Electrocardiol 1996;29(Suppl):21-5.

Address for correspondence.

Jocelyne Fayn.

INSERM ERM107, Hôpital cardiologique, BP lyon Montchat, 69394 LYON cedex 03, France.

E-mail: fayn@insa.insa-lyon.fr. 